\title{
PROJECTIVE GEOMETRY ON PARTIALLY ORDERED SETS
}

\author{
BY
}

\author{
ULRICH FAIGLE AND CHRISTIAN HERRMANN
}

\begin{abstract}
A set of axioms is presented for a projective geometry as an incidence structure on partially ordered sets of "points" and "lines". The axioms reduce to the axioms of classical projective geometry in the case where the points and lines are unordered. It is shown that the lattice of linear subsets of a projective geometry is modular and that every modular lattice of finite length is isomorphic to the lattice of linear subsets of some finite-dimensional projective geometry. Primary geometries are introduced as the incidence-geometric counterpart of primary lattices. Thus the theory of finite-dimensional projective geometries includes the theory of finite-dimensional projective Hjelmslev-spaces of level $\boldsymbol{n}$ as a special case. Finally, projective geometries are characterized by incidence properties of points and hyperplanes.
\end{abstract}

1. Introduction. Veblen and Young's [20] classical approach to projective geometry views a projective space as an incidence structure involving "points" and "lines" subject to certain requirements. If a projective space is finite dimensional, its lattice of linear subsets is modular, complemented, and of finite length. In fact, every modular complemented lattice of finite length may (up to isomorphism, of course) be understood as the lattice of linear subsets of a unique finite-dimensional projective space (see Birkhoff [3, p. 93]). A third aspect of projective geometry derives from the fact that a projective space may be coordinatized over some (skew) field provided its incidence structure is rich enough. In this case the lattice of linear subsets essentially is the lattice of subspaces of some vector space over the coordinatizing field.

Baer [2] took the latter observation as a starting point for widening the scope of projective geometry: he studied the lattice of submodules of a finitely generated module over a completely primary uniserial ring and obtained a theory which includes the theory of classical projective geometry and the theory of finite abelian groups as special cases (see also, e.g., Inaba [9], Ribeiro [19], Jónsson and Monk [10]).

Thereby it was noted that not just the atoms but also certain other join-irreducible elements of a primary lattice behave like points of a projective geometry. On the other hand, Kurinnoi [14] observed that two finite modular lattices are isomorphic if and only if their partially ordered sets of all elements that are joins of at most two join-irreducible elements are isomorphic. For complemented modular

Received by the editors June 4, 1980. Presented at the Oberwolfach meeting on "Combinatorics of ordered sets", May 18-24, 1980.

1980 Mathematics Subject Classification. Primary 51A05; Secondary 06 C05.

Key words and phrases. Point, line, incidence structure, projective geometry, modular lattice. 
lattices, Kurinnoi's result follows immediately from the fact that a projective space is determined by the incidence structure of its points and lines.

Thus the question arises: Does there exist a theory of projective incidence geometry which, in particular, allows us to understand the set of all join-irreducible elements of a modular lattice of finite length as the set of points of some finite-dimensional projective space?

We present a system of axioms for such a projective geometry on partially ordered sets of points and lines in $\$ 3$. The crucial property of a classical projective incidence geometry is expressed in Veblen and Young's "triangle axiom". In the general context, however, one quickly recognizes that the triangle axiom in its usual form is too restrictive to be satisfied. It is therefore surprising that a general projective incidence geometry does have a property which reduces to the classical triangle axiom in the case of unordered points and lines. This is stated in our axiom (A7). We then show in $\$ 4$ that there is a correspondence between projective geometries and modular lattices of finite length in the same way as between classical projective geometries and complemented modular lattices of finite length. In $\$ 5$ primary geometries are introduced as the incidence-geometric counterpart of primary lattices. Generalizations of projective incidence geometries have been looked at before: projective Hjelmslev spaces may be seen that way (see, e.g., Klingenberg [12], [13], Lück [15], and Artmann [1]). As an example, we will indicate in $\$ 6$ how projective Hjelmslev planes of level $n$ can be interpreted as special instances of our primary geometries. Finally, in $\$ 7$, we will characterize projective geometries by incidence properties of points and hyperplanes. Those properties may be taken as axioms for a theory of "subprojective geometries" in the sense of Markowsky and Petrich [16].

In passing we mention the connection between the projective geometries introduced here and the geometries on partially ordered sets of Faigle [8]. The latter extend the concept of a matroid from unordered ground sets to partially ordered sets. Thus, in the same way as a matroid may be thought of as a generalization of a projective geometry, those geometries on partially ordered sets are generalizations of projective geometries on partially ordered sets.

2. Preliminaries. This section collects some basic definitions and properties of partially ordered sets, especially modular lattices, that we will use in the sequel without further reference. Proofs of the statements can be found in Birkhoff [3] or Crawley and Dilworth [4].

A chain of a partially ordered set $P$ is a subset of pairwise comparable elements of $P$. The length of a chain is its cardinality minus one. By the height $r(p)$ of an element $p \in P$ we understand the length of the longest chain in $P$ with maximal element $p$. This number is well defined if $P$ is of finite length, i.e., if the lengths of the chains of $P$ are bound by some natural number. If $P$ in particular is a lattice, we will use the term "rank" instead of "height". $r(P)=\max \{r(p): p \in P\}$ is the height (rank) of $P$.

An (order) ideal of $P$ is a subset $A$ with the property that $p<q$ implies $p \in A$ whenever $q \in A$. 
The element $y$ covers the element $x$ in $P$ if the interval $[x, y]=\{z \in P$ : $x<z<y$ \} of $P$ consists of just the two elements $x$ and $y$. We denote this by $x \prec y$.

The dual of $P$ is the set $P$ with the inverted order.

If the lattice $M$ is of finite length, $M$ is in particular complete, i.e., join and meet of every subset of $M$ exist. So $M$ has a maximal element 1 and a minimal element 0 . Moreover, every element of $M$ is a join of finitely many join-irreducible elements and, dually, a meet of finitely many meet-irreducible elements. Here we call an element $x \neq 0$ join-irreducible if $x=y \vee z$ always implies $x=y$ or $x=z$. Meetirreducible elements are defined dually.

A lattice $M$ is modular if for all $x, y, z \in M, x<z$ implies $(x+y) z=x+y z$. Note that in a modular lattice we use the notation $x+y(x y)$ for the join (meet) of the elements $x$ and $y$.

A modular lattice $M$ of finite length is also characterized by a property of the rank function:

$$
\text { for all } x, y \in M, \quad r(x+y)+r(x y)=r(x)+r(y) \text {. }
$$

Two intervals of a modular lattice of the form $[a b, b]$ and $[a, a+b]$ are projective. Projective intervals always are isomorphic.

In a modular lattice of finite length, the number of elements in any irredundant representation of the element $x$ by join-irreducible elements is unique by the theorem of Kuroš and Ore. We call this number the (Kuroš-Ore) dimension $d(x)$ of $x$. Thus $x$ is join-irreducible if $d(x)=1$. If furthermore $r(x)=1, x$ is an atom.

We finally prove two useful facts about modular lattices.

LEMMA 2.1. Let $M$ be any modular lattice and $p, q, p<q$, join-irreducible elements of $M$. Then $q \leqslant x+p$ implies $q \leqslant x$ for all $x \in M$.

Proof. Since $M$ is modular, $q=(p+x) q=p+x q$. Hence $q=x q$ because $q$ was join-irreducible.

LEMMA 2.2. Let $M$ be any modular lattice and $C$ any maximal chain of $M$. Then the length of $M$ equals the length of $C$ when $C$ is of finite length.

Proof. Proof of [3, Theorem 14, p. 40].

3. Projective incidence geometries on partially ordered sets. We give in this section a set of axioms for a projective geometry whose points and lines are partially ordered, and we derive the fundamental properties of the lattice of linear subsets of such a geometry. In the special case of trivially ordered sets those axioms are easily seen to be equivalent to the axioms of classical incidence geometry as given by Veblen and Young [20]. Note that, in general, a line need not be incident with at least three points. We will, however, study an important class of geometries satisfying the analogue of the latter property, the primary geometries, in $\$ 5$.

From the outset we impose a finiteness condition on the geometry to be defined and assume that the partially ordered set of points is of finite length. What happens without any finiteness condition will be briefly outlined at the end of $\S 4$. 
The symbol " $\in$ " is used both with the usual meaning and with the understanding of an incidence relation between points and lines. This should cause no confusion.

Let $P$ and $L$ be two disjoint partially ordered sets of points and lines, respectively, and $\in \subset P \times L$ an incidence relation between points and lines such that the following axioms hold:

(A1) For $p \leqslant q \in P, g \in L, q \in g$ implies $p \in g$. For $g<m \in L, p \in P, p \in g$ implies $p \in m$.

(A2) Any two noncomparable points $p$ and $q$ are incident with a unique line, denoted $p \vee q$, such that for every line $g, p, q \in g$ implies $p \vee q<g$.

(A3) Every line $g$ is of the form $g=p \vee q$ for some noncomparable points $p$ and $q$.

(A4) If $p \vee q$ is a line and $p^{\prime}>p$ a point, $p^{\prime} \notin p \vee q$.

(A5) If $p \vee q$ is a line and $r$ a point so that $p, q \leq r$, then $t<r$ for all $t \in p \vee q$. $G=(P, L, \in)$ is then an incidence geometry on $P$ and $L$.

In view of (A2), every line of $G$ may be identified with the ideal of all points incident with it. Furthermore, two comparable points $p<q$ may be taken to generate the degenerate line $p \vee q$, which we view as the ideal $\{r \in P: r<q\}$. Thus the set of degenerate lines, ordered by containment, is isomorphic to the set of points of $G$. We will make use of those identifications whenever convenient.

A linear set of $G$ is a set $S$ of points such that for every $p, q \in S, p \vee q \subset S$. So each (possibly degenerate) line is a linear set and $P$ itself is linear. The intersection $\bigwedge S_{i}$ of linear sets $S_{i}$ is linear. Defining the join $\bigvee S_{i}$ as the smallest linear set containing all $S_{i}$, we see that the collection $M(G)$ of linear sets of $G$ forms a complete lattice. Moreover, calling an element $p \neq 0$ of a lattice $M$ completely join-irreducible if $p=\bigvee T$ implies $p=t$ for some $t \in T$, for all $T \subset M$, we note

Proposition 3.1. Let $G=(P, L, \in)$ be an incidence geometry. Then $P$ is isomorphic to the set of completely join-irreducible elements of $M(G)$.

Proof. Since every linear set of $G$ is a union of degenerate lines, a completely join-irreducible element of $M(G)$ must be a degenerate line.

Conversely, if $p$ is a point of $G$, let $(p)=\cup\{r \vee q: r, q<p\}$. By axiom (A5), $(p)$ is a linear set. Moreover, $(p)$ is the join of all linear sets properly contained in $p \vee p$. By axiom (A4), $p \notin(p)$. Hence the degenerate line $p \vee p$ must be completely join-irreducible.

Note that a line of an incidence geometry is not necessarily generated by just any two noncomparable points incident with it. We call the incidence geometry $G$ line-regular if $G$ satisfies the following weak analogue of this property:

(A6) If $p, q, r$ are pairwise noncomparable points such that $r \in p \vee q$, then there exists a point $p^{\prime} \leqslant p$ so that $p^{\prime} \vee q=r \vee q$.

We now come to the central definition.

A projective geometry is a line-regular incidence geometry $P G=(P, L, \in)$ satisfying: 
(A7) If $a, p, c, q, b$ are points such that $c \in a \vee p$ and $q \in b \vee c$, then there exists a point $x \in a \vee b$ so that $q \in p \vee x$.

This "triangle property" is illustrated in Figure 1:

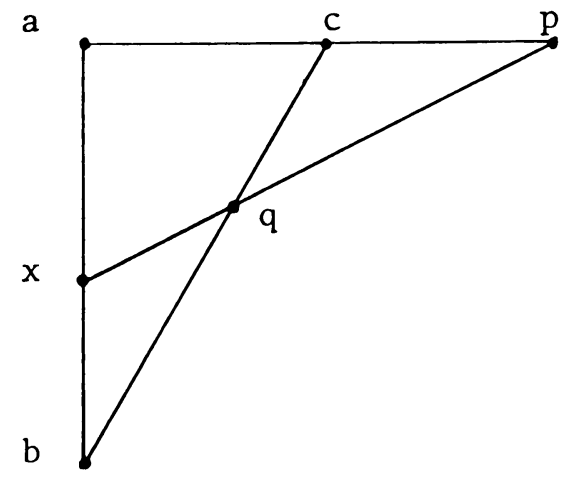

Figure 1

In the statement of axiom (A7) we did not explicitly require the lines $a \vee p$, $b \vee c$, or $a \vee b$ to be nondegenerate. That this is only a matter of convenience follows from the next lemma.

LEMMA 3.2. Let $a, p, c, q, b$ be points of $a$ (not necessarily projective) incidence geometry such that $a \vee p, b \vee c$, or $a \vee b$ is degenerate. Then the conclusion of axiom (A7) holds.

Proof. Suppose, for instance, $a \leqslant p$. Then $c \leqslant p$. Hence $q \in b \vee c$ implies $q \in b \vee p$.

If $a \geqslant p$, then $c \leqslant a$. Hence $q \in a \vee b$.

The other cases are checked as easily.

Many properties of linear sets of classical projective geometries now carry over to the general case as we will proceed to show.

Proposition 3.3. Let $S$ and $T$ be two linear sets of a projective geometry $P G=(P, L, \in)$. Then

$$
S \vee T=\{r \in P: r \in p \vee q \text { for some } p, q \in S \cup T\}
$$

Proof. It suffices to show that the set in the statement of the proposition is linear. So let $a, b, c, d, p, q, r$ be points of $P G$ such that $a, b \in S, c, d \in T$, $p \in a \vee c, q \in b \vee d$, and $r \in p \vee q$. We must show that there exist points $s \in S$, $t \in T$ such that $r \in s \vee t$.

Applying axiom (A7) to the points $a, c, r, q$, we obtain a point $u \in a \vee q$ so that $r \in c \vee u$. So we can apply (A7) to $b, d, q, u, a$ and get a point $s \in a \vee b$ so that $u \in d \bigvee s$. Finally, using (A7) with the points $d, s, u, r, c$, we conclude the existence of a point $t \in d \vee c$ such that $r \in s \vee t$ (see Figure 2). 


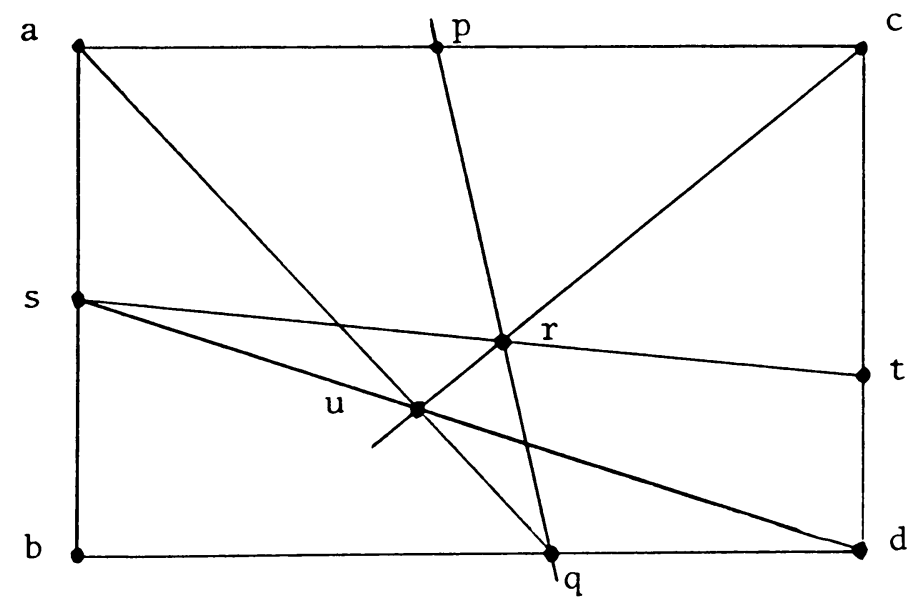

FIgURE 2

Proposition 3.4. The lattice $M(P G)$ of linear sets of a projective geometry $P G$ is modular.

Proof. If $X, Y, Z$ are arbitrary linear sets such that $X \subset Z$, we must show $X \vee(Y \wedge Z) \supset(X \vee Y) \wedge Z$ since the inequality $X \vee(Y \wedge Z) \subset(X \vee Y) \wedge$ $Z$ holds in any lattice.

$X \vee(Y \wedge Z)$ consists of all points of $X \cup(Y \wedge Z)$ and all points incident with some line between $X$ and $Y \wedge Z$ (Proposition 3.3).

Let $p \in(X \vee Y) \wedge Z$. W.l.o.g., we may assume $p \notin X \cup(Y \wedge Z)$. So $p \in x \vee$ $y$ for some $x \in X, y \in Y$. Since $P G$ is line-regular, there is a point $y^{\prime}<y$ so that $p \vee x=x \vee y^{\prime}$, i.e., $y^{\prime} \in Y \wedge Z$ because $p, x \in Z$. Hence $p$ is incident with a line between $X$ and $Y \wedge Z$.

Consider the statement

(A8) For every linear set $S$ there exists a finite set $B$ of points such that $S$ is the smallest linear set containing $B$.

Proposition 3.5. The lattice $M(P G)$ of linear sets of the projective geometry $P G$ is of finite length iff (A8) holds for $P G$.

Proof. In a lattice of finite length an element is completely join-irreducible iff it is join-irreducible. Hence the necessity of (A8) follows from Proposition 3.1.

To show sufficiency, we first claim that if $\bar{p}=p \vee p$ is a degenerate line of $P G$, then the interval $[\varnothing, \bar{p}]$ is of finite length.

Suppose this is not the case and let $p \in P$ be a minimal (with respect to the height in $P$ ) counterexample. Since $\bar{p}$ is completely join-irreducible (Proposition 3.1), it has a unique lower neighbor $(p) \prec \bar{p}$ in $M(P G)$. By (A8), there are points $p_{i}<p, i=1, \ldots, n$, such that $(p)=\bar{p}_{1} \vee \cdots \vee \bar{p}_{n}$. By the choice of $p$, every interval $\left[\varnothing, \bar{p}_{i}\right]$ is of finite length. So, in particular, every $\bar{p}_{i}$ is of finite rank in $M(P G)$. Consequently, using the remark of $[4, p .27],(p)$ is of finite rank in $M(P G)$. But this means that $\bar{p}$ is of finite rank.

The general case then follows by the same argument. 
A projective geometry $P G$ with the property (A8) is finite dimensional. Proposition 3.5 allows us to assign to every linear set $S$ of $P G$ the (Kuroš-Ore) dimension $d(S)$, namely the dimension of $S$ in the lattice $M(P G) . d(P G)=d(P)$ is the dimension of $P G$. So the dimension of a linear set is just the minimal number of points needed to span this linear set.

We end this section by giving two examples of projective geometries on partially ordered sets.

EXAMPLE 3.6. With a finite abelian group $A$ a projective geometry $P G(A)$ may be associated in the following way. The points of $P G(A)$ are the cyclic subgroups of $A$ of prime power order. The lines of $P G(A)$ are the subgroups of $A$ that can be represented as direct sums of two points. The incidence structure is then given by containment.

EXAMPLE 3.7. A projective Hjelmslev plane of level $\boldsymbol{n}$ is an incidence structure with neighborhood on unordered sets of points and lines. It turns out, however, that a projective Hjelmslev plane of level $n$ corresponds in a very natural way to a projective geometry on partially ordered sets of points and lines where no concept of neighborhood is needed. We give more details in $§ 6$.

In both examples, the lattices of linear sets are cyclic in the sense of $\$ 5$. The partially ordered sets of points therefore exhibit a special structure: their Hasse diagram is a disjoint union of rooted trees whose roots correspond to the minimal points.

4. The geometry of a modular lattice. It was shown in the preceding section that every finite-dimensional projective geometry gives rise to a modular lattice of finite length. In this section we will associate with each modular lattice of finite length a projective geometry in a natural way so that the lattice of linear sets thereby obtained is isomorphic to the lattice we started out with. This means that a modular lattice of finite length essentially is the lattice of linear sets of some projective geometry.

So let $M$ be a modular lattice of finite length. By $P G(M)$ we understand the sets $P=\{p \in M: d(p)=1\}$ and $L=\{g \in M: d(g)=2\}$ together with the order and incidence relation implied by $M$. As before, we refer to $P$ and $L$ as sets of points and lines of $P G(M)$.

Proposition 4.1. $P G(M)$ is an incidence geometry.

Proof. (A1), (A2), and (A5) are obvious. (A3) is just the definition of the dimension of a line. (A4) is a direct consequence of Lemma 2.1.

In order to distinguish more clearly between the lattice-theoretic concepts of $M$ and the geometric concepts of the lattice $M(P G)$ of linear sets of $P G=P G(M)$, it is convenient to introduce a new notation: for $T \subset P$, let

$$
\bar{T}=\bigwedge\{S \in M(P G): T \subset S\}
$$

Thus $T \in M(P G)$ iff $\bar{T}=T$ iff $p \vee q \subset T$ for all $p, q \in T$.

Our goal is to show that $P G$ is a projective geometry and that $M(P G)$ is isomorphic to $M$. 
THEOREM 4.2. Let $x, y \in M, z \in P$ be arbitrary elements so that $z \$ y$ but $z \leqslant x+y$. Then there exists $x^{\prime} \leqslant x, x^{\prime} \in P$, with the property $x^{\prime}+y=z+y$.

Proof. W.l.o.g., we may assume $z+y=x+y$ (otherwise we replace $x$ by $x(y+z))$. With $\bar{z}=x z+y z$ and $\bar{x}=z x+y x$, we compute $\bar{z}+(\bar{x}+y)=\bar{x}+$ $y, z+(\bar{x}+y)=z+y=x+y$, and $(\bar{x}+y) z=(\bar{x} z+y) z=\bar{x} z+y z=\bar{z}$, $(x+y) z=z$. Thus $[\bar{z}, z]$ and $[\bar{x}+y, x+y]$ and, similarly, $[\bar{x}, x]$ and $[\bar{x}+y, x+$ $y]$ are projective. Hence $[\bar{x}, x]$ and $[\bar{z}, z]$ are isomorphic.

If $\bar{z}=z$, then $z \in P$ implies $z=x z$, i.e., $z \leqslant x$, and we may take $x^{\prime}=z$.

If $\bar{z}<z, z$ has a unique lower neighbor $\underline{z}, \bar{z} \leqslant \underline{z} \prec z$. Let $\underline{x}$ be the unique lower neighbor of $x$ in $[\bar{x}, x]$. Since every element of $M$ is a join of members of $P$, there exists $x^{\prime} \in P$ such that $x^{\prime} \leqslant x$ and $x^{\prime} \nless \underline{x}$. So $x^{\prime}+\bar{x} \$ \underline{x}$ allows us to conclude $x^{\prime}+\bar{x}=x$. Furthermore, $\underline{z}+y \geqslant \bar{z}+y \geqslant \bar{x}$. Therefore $\underline{z}+x^{\prime}+y>x^{\prime}+\bar{x}+$ $y=x+y$. Finally, $\underline{z}<z$ implies $\underline{z}+\left(x^{\prime}+y\right) z=\left(\underline{z}+x^{\prime}+y\right) z=z$, i.e., $z<x^{\prime}$ $+y$ since $z \in P$. Hence $x^{\prime}+y=z+y$.

COROLlary 4.3. PG is a projective geometry.

Proof. Property (A6) is a special case of Theorem 4.2.

For (A7), let $x=a+b, y=p$, and $z=q$.

LeMma 4.4. For $X \subset P, \Sigma \bar{X}=\Sigma X$.

Proof. The lemma follows immediately from the observation

$$
\bar{X}=\bigcup\{p \vee q: p, q \in X\}
$$

Lemma 4.5. If $T$ is a linear set of $P G$ and $p \in P$ is such that $p<\Sigma T$, then $p \in T$.

Proof. Since $M$ is of finite length, there are elements $p_{i} \in T, i=1, \ldots, n$, such that $p<p_{1}+\cdots+p_{n}$. We use induction on $n$.

The case $n \leqslant 2$ follows directly from the definition.

Let $T^{\prime}=\left\{\overline{p_{1}, \ldots, p_{n}}\right\}$. By Lemma 4.4, $p<\Sigma T^{\prime}+p_{n}$. Hence, by Theorem 4.2, there is $q \in P, q \leqslant \Sigma T^{\prime}$, with $p \leqslant q+p_{n}$. By the induction hypothesis, $q \in T^{\prime}$, i.e., $p \in\left\{\overline{T^{\prime}, p_{n}}\right\} \subset T$.

Lemmas 4.4 and 4.5 together yield as a consequence that the map $T \rightarrow \Sigma T$ is an isomorphism from $M(P G)$ onto $M$. We can therefore summarize:

THEOREM 4.6. Every modular lattice $M$ of finite length is isomorphic with the lattice $M(P G)$ of linear sets of the projective geometry $P G(M)$ naturally associated with $M$.

Corollary 4.7 (Kurinnoi [14]). Let $M$ and $M^{\prime}$ be modular lattices of finite length. Then $M$ and $M^{\prime}$ are isomorphic iff their partially ordered sets of elements that are joins of at most two join-irreducible elements are isomorphic.

Another immediate consequence is the "Verbindungssatz":

COROllary 4.8. Let $M$ be a modular lattice of finite length and $p<a+b$ in $M, p$ join-irreducible. Then there are join-irreducible elements $a^{\prime}<a$ and $b^{\prime}<b$ in $M$ such that $p<a^{\prime}+b^{\prime}$. 
The application is a test for lattice identities which is well known in the case of complemented modular lattices.

COROLlARY 4.9. Let $w$ and $w^{\prime}$ be lattice words such that in $w$ no variable appears more than once. Then the inequality $w \leqslant w^{\prime}$ is valid in a given modular lattice $M$ of finite length provided it is valid for every substitution of join-irreducibles for the variables.

Examples of such inequalities are the Arguesian law of Jónsson and Monk [10], the dual $n$-distributive inequality of A. Huhn (related to dimension), and the Fano inequality of Wille [21]. Inequalities of this type expressing other characteristics of coordinatizing rings can also be constructed.

The infinite case. In the derivation of the results of $\$ 3$ no use was made of any finiteness condition imposed on the projective geometry $P G$ except in the proof of Theorem 3.5. Dropping the assumption that the set $P$ of points of $P G$ be of finite length and not requiring property (A8) to hold for $P G$, one may therefore ask whether a general analogue of Theorem 4.6 can be obtained. It turns out that a general projective geometry will have a modular lattice of linear sets which is algebraic such that every element is the join of compact irreducible elements. In this context, the analogue of Theorem 4.6 holds. This may be proved exactly along the lines of the proofs given so far.

For another approach to a theory of an infinite analogue of projetive geometries of finite dimension see $\$ 7$.

5. Primary geometries. So far we have outlined a theory of projective incidence geometry that encompasses the theory of modular lattices (of finite length). At this level of generality one cannot expect too many features of classical projective geometry to reveal themselves. We will therefore first characterize the projective geometries whose dimension function is monotone in the sense that $S \subset T$ implies $d(S) \leqslant d(T)$ for any two linear sets $S$ and $T$. We will then exhibit the projective geometries that have the additional property that every line of any minor is incident with at least three minimal points (by a minor of a projective geometry $P G$ we understand the projective geometry associated with an interval of $M(P G)$ ). Those are the primary geometries. Primary geometries admit a theory of coordinatization similar to the classical theory: the role of the coordinatizing field is taken over by a completely primary uniserial ring (see, e.g., Jónsson and Monk [10] for details).

We will throughout assume all projective geometries to be finite dimensional and all lattices to be of finite length.

A cycle of a lattice $M$ is a nonzero element $z \in M$ such that $[0, z]$ is a chain. $M$ is cyclic if every element of $M$ is a join of cycles, i.e., $M$ is cyclic precisely when the set of join-irreducible elements is the set of cycles.

THeOREM 5.1. Let $M$ be a modular lattice. Then $M$ is cyclic iff for every $x, y \in M$, $x \leqslant y$ implies $d(x) \leqslant d(y)$. 
Proof. Assume that $M$ is not cyclic. Then there is an element $p \in P$, the set of points of $P G(M)$, which is not a cycle. Hence $[0, p]$ contains at least two noncomparable elements $q, r \in P$. So we have $q+r<p$ and $d(q+r)>d(p)$.

Conversely, assume that $M$ is cyclic.

Note first that for every $p \in P, a \in M, p \nless a$ implies that $a+p$ is join-irreducible in $[a, 1]$. This follows from the isomorphism of $[p a, p]$ and $[a, p+a]$. Consequently, if $[a, b]$ is any interval of $M$ and $x \in[a, b]$, the dimension of $x$ with respect to $[a, b]$ does not exceed $d(x)$.

Suppose that the theorem fails to hold. Then we choose $x, y \in M, y$ of minimal rank such that $x \leqslant y$ and $n=d(x)>d(y)=m$. So we may write $y=p+u$ for suitable $p \in P, u \in M$ with $d(u)=m-1$. Similarly, $x=x_{1}+\cdots+x_{n}$ for suitable elements $x_{i} \in P$.

By Theorem 4.2, there are elements $p_{i}, 0<p_{i}<p$, such that $x_{i}+u=p_{i}+u$, $i=1, \ldots, n$. Moreover, since $p$ is a cycle, all $p_{i}$ 's are comparable. So, w.l.o.g., $p_{n}+u=p_{1}+\cdots+p_{n}+u=x_{1}+\cdots+x_{n}+u=x+u$.

Hence $x \in\left[x_{n}, p_{n}+u\right]$, and the dimension of $x$ with respect to $\left[x_{n}, p_{n}+u\right]$ must be equal to $n-1$. Since $\left[x_{n}, p_{n}+u\right]$ and $\left[x_{n} p_{n}, u\right]$ are isomorphic, $[0, u]$ must contain an element of dimension at least $n-1$ by the remark above, a contradiction to $d(u)=m-1<n-1$ and the choice of $y$.

A cyclic modular lattice $M$ is $k$-primary $(2<k<\infty)$ if every interval of $M$ which is not a chain has at least $k$ atoms. The next proposition says that $k$-primarity really is a property of the lines of the projective geometry $P G(M)$.

Proposition 5.2. A cyclic modular lattice $M$ is $k$-primary iff for all $p, q \in P$, $[0, p+q]$ is k-primary.

Proof. Let $[x, y]$ be an interval of $M$ which is not a chain. Then we may choose $a, b \in M$ such that $a b$ is of minimal rank with the property $x<a b<a, b<a+$ $b<y$.

Let $a^{\prime}, b^{\prime} \in P$ be such that $a=a^{\prime}+a b$ and $b=b^{\prime}+a b$. We may assume $a=a^{\prime}+x$ and $b=b^{\prime}+x$ since $a b$ was chosen minimal. Thus $a+b=x+a^{\prime}+$ $b^{\prime}$. Hence $[x, a+b]$ and $\left[x\left(a^{\prime}+b^{\prime}\right), a^{\prime}+b^{\prime}\right]$ are isomorphic.

Consequently the interval $[x, a+b]$ and, a fortiori, $[x, y]$ must contain at least $k$ atoms.

A note on the terminology: 2-primary lattices are also called semiprimary whereas 3-primary lattices are simply termed primary (cf. [10]).

We will take a closer look at semiprimary lattices. By Proposition 5.2, it suffices to restrict our attention to the case of dimension 2 .

Proposition 5.3. Let $M$ be a semiprimary lattice, $d(M)=2$. Then if $z \in P$ is $a$ maximal cycle of $M,[z, 1]$ is a chain.

Proof. If not, there are atoms $g \neq m \in[z, 1]$ such that $z<g, m \prec g+m$. Choose $a, b \in P$ so that $g=z+a$ and $m=z+b$.

Since $M$ is semiprimary, there is an atom $c \in P$ with $c \$ z$. Hence we may assume $g=z+c$. 
Now $z \leqslant c+b$ is impossible because $b<m$ and $r(c+b)<r(b)+1$. Also, $c \leqslant z+b$ and $b \leqslant z+c$ are impossible since $g$ and $m$ are noncomparable.

Hence $d(z+b+c)=3$, contradicting $d(M)=2$.

Proposition 5.4. Let $M$ be a cyclic modular lattice such that for all noncomparable cycles $p, q \in P,[0, p+q]$ contains at least 2 atoms and $[z, p+q]$ is a chain whenever $z$ is a maximal cycle in $[0, p+q]$. Then $M$ is semiprimary.

Proof. If not, there is an interval $[x, y]$ in $M$ which is not semiprimary. Choose $y$ of minimal rank with this property. By Proposition 5.2, $d(y)=2$.

By the hypothesis on $M, x$ cannot be a maximal cycle in $[0, y]$.

If $x$ is a cycle, there is a cycle $x^{\prime}>x$. Moreover, there is an atom $c \in[0, y]$ with $c \nless x$. Hence $c+x>x$ and $x^{\prime} \neq c+x$. So $[x, y]$ contains two atoms.

If $x$ is not a cycle, then $x=p+q$ for some noncomparable cycles $p, q \in P$. Neither $p$ nor $q$ can be a maximal cycle since otherwise $[x, y]$ is a chain according to the hypothesis on $M$.

Let $p^{\prime}, q^{\prime}$ be cycles such that $p^{\prime}>p$ and $q^{\prime}>q$. Then $p^{\prime} \nless p+q^{\prime}$ and $q^{\prime} \nless p^{\prime}+$ $q$ by Lemma 2.1. Hence $p^{\prime}+q \neq p+q^{\prime}$ and $x<p+q^{\prime}, p^{\prime}+q$ implies that $[x, y]$ contains at least two atoms.

We define a finite-dimensional projective geometry $P G=(P, L, \in)$ to be semiprimary if every degenerate line of $P G$ is a chain and $P G$ satisfies the following axiom:

(SP) Every line is incident with at least two minimal points. Moreover, if $g, m, h$ are lines such that $g, m \leqslant h$ and both $g$ and $m$ are incident with a maximal point of $h$, then $g \leqslant m$ or $m \leqslant g$.

From Propositions 5.3 and 5.4 we then obtain

THEOREM 5.5. A projective geometry is semiprimary iff its lattice of linear sets is semiprimary.

The semiprimary geometry $P G=(P, L, \in)$ is called primary if:

(P) For every two noncomparable points $p$ and $q$, there exists a point $r$ such that $p \vee q=p \vee r=r \vee q$.

THEOREM 5.6. A projective geometry $P G$ is primary iff its lattice $M(P G)$ of linear sets is primary.

Proof. It suffices to verify the theorem for projective geometries $P G$ with dimension $d(P G)=2$. So assume that $P G$ is primary but $M(P G)$ is not primary. Then there are $x, y \in M(P G)$ such that $[x, y]$ contains exactly two atoms $a$ and $b$. By induction on the rank of $M(P G)$, we may assume $a+b=y=1$. Also, there are $a^{\prime}, b^{\prime} \in P$ such that $a=x+a^{\prime}$ and $b=x+b^{\prime}$.

If $a^{\prime}+b^{\prime}<y$, consider $\left[x, x+a^{\prime}+b^{\prime}\right]$ and $\left[x\left(a^{\prime}+b^{\prime}\right), a^{\prime}+b^{\prime}\right]$, the isomorphic intervals, and conclude that $\left[x, x+a^{\prime}+b^{\prime}\right]$ must contain at least three atoms, a contradiction.

If $a^{\prime}+b^{\prime}=y$, choose $r \in P$ such that $a^{\prime}+b^{\prime}=a^{\prime}+r=b^{\prime}+r$. Since $r$ is a cycle, $[x, x+r]$ must be a cycle because $[r x, r]$ and $[x, x+r]$ are isomorphic. 
$x+r \leqslant a, b$ is impossible since $r+a=r+b=y$.

Thus $x+r$ must be a third atom of $[x, a+b]$.

Conversely, we must show that for all noncomparable cycles $a, b$ of a primary lattice $M$, there is a cycle $r$ such that $a+b=a+r=b+r$.

Note first that an element $z \in[x, y]$ which is join-irreducible in the interval $[x, y]$ must be of the form $z=x+p$ for some join-irreducible element $p$ of $M$. This may be seen from the representation $z=x+z=x+\left(p_{1}+\cdots+p_{n}\right)=(x$ $\left.+p_{1}\right)+\cdots+\left(x+p_{n}\right)$.

We now proceed by induction on the rank.

If $a$ and $b$ are atoms of $M$, the existence of $r$ is a direct consequence of the definition of a primary lattice.

For the induction step, assume that $a$ is not an atom and let $a^{\prime}$ be a cycle strictly below $a$. Then $a^{\prime}+b$ is a cycle in $\left[a^{\prime}, a+b\right]$. Moreover, $a \$ a^{\prime}+b$ (Lemma 2.1) and $a^{\prime}+b \$ a$. Because $r\left(\left[a^{\prime}, a+b\right]\right)<r([0, a+b])$, we conclude therefore the existence of a cycle $r$ of $M$ such that $a+b=a+\left(a^{\prime}+b\right)=a+\left(a^{\prime}+r\right)=a+$ $r=a^{\prime}+b+r$. By Lemma 2.1, we must have $a \leqslant b+r$, i.e., $a+r=b+r$.

Jónsson and Monk [10] have shown that every primary lattice $M$ which is not a chain is simple. Furthermore, if $d(M) \geqslant 3$, any two intervals of length 2 that are not chains are isomorphic [10, Theorem 6.3]. Consequently, calling a primary geometry $k$-primary if every line is incident with at least $k$ minimal points, we may state:

THEORem 5.7. A primary geometry $P G$ of dimension $d(P G)>3$ is $k$-primary iff $M(P G)$ is a k-primary lattice.

6. Example: Projective Hjelmslev planes of level $\boldsymbol{n}$. It is well known that every element of a semiprimary lattice $M$ may be written as a sum of independent cycles (see, e.g., [10]), where the cycles $p_{1}, \ldots, p_{n}$ of $M$ are said to be independent if $r\left(p_{1}+\cdots+p_{n}\right)=r\left(p_{1}\right)+\cdots+r\left(p_{n}\right)$. If the element $1 \in M$ can be written as sum of independent cycles all having the same rank $n, M$ is n-homogeneous. It follows from [10, Theorem 5.3] that all maximal cycles of an $n$-homogeneous semiprimary lattice have the same rank.

$n$-homogeneous primary lattices $M$ of dimension $d(M)=3$ have been looked at from an incidence-geometric point of view by Artmann [1], who suggested Hjelmslev spaces as defined by Klingenberg [12], [13] and Lück [15] as the appropriate analogue of classical projective spaces. In particular, he showed that with each $n$-homogeneous primary lattice $M, d(M)=3$, a projective Hjelmslev plane $H(M)$ of level $\boldsymbol{n}$ ( $\boldsymbol{n}$ - $\boldsymbol{H}$-plane) may be associated in the following way:

The points of $H(M)$ are the maximal cycles of $M$ and the lines of $H(M)$ the joins of two independent points. The incidence relation of $H(M)$ is the incidence relation implied by $M$.

Dugas [6] then proved the converse: any $n-H$-plane $H$ is isomorphic to the associated $n$ - $H$-plane $H(M)$ of some (up to isomorphism) unique $n$-homogeneous primary lattice $M$ with $d(M)=3$.

In our terminology, a $n$ - $H$-plane therefore essentially is a primary geometry of dimension three where all maximal points share the same rank. 
We believe that this approach to projective Hjelmslev planes of level $n$ offers some advantage. We do not need any concept of "neighborhood" among points. Moreover, in the arguesian case, our linear sets correspond to all submodules of some module $R^{3}$ over a completely primary and uniserial ring $R$, which seems to be more natural than the consideration of only the direct factors of $R^{3}$.

7. Point-hyperplane incidence. Classical projective spaces can be characterized by a set of self-dual axioms involving just the incidence relation between points and hyperplanes (see, e.g., Esser [7], Dembowsky and Wagner [5], Kantor [11], and Markowsky and Petrich [16]).

In this section we offer such a set of axioms for projective geometries on partially ordered sets. A hyperplane in this context will, of course, mean the dual of a point, namely a linear set which is meet-irreducible in the lattice of linear sets.

Let $P$ and $H$ be distinct partially ordered sets and $I \subset P \times H$ an incidence relation such that

$\left(\mathrm{S}_{1}\right)$ For all $p \leqslant q \in P, h \in H, q I h$ implies $p I h$.

$\left(\mathrm{S}_{1}^{\prime}\right)$ For all $g \leqslant h \in H, p \in P, p I g$ implies $p I h$.

To avoid trivialities, we will assume that none of the following sets is empty:

for $p \in P, p^{\prime}=\{h \in H: p I h\}$ and, dually,

for $h \in H, h^{\prime \prime}=\{p \in P: p I h\}$.

A subset $S \subset P(H)$ is closed if $S$ is an intersection of sets of the form $h^{\prime \prime}\left(p^{\prime}\right)$. Thus we obtain the (complete) lattice $M(P)(M(H))$ of closed subsets of $P(H)$.

From the theory of Galois connections (see, e.g., Ore [18]) one easily deduces that $M(P)$ and $M(H)$ are dually isomorphic via the Galois connection:

for $S \subset P, S \mapsto\{h \in H: s I h$ for all $s \in S\}$ and

for $T \subset H, T \mapsto\{p \in P: p I t$ for all $t \in T\}$.

We next assume that the incidence structure $(P, H, I)$ satisfies, in addition:

$\left(\mathrm{S}_{2}\right)$ For all $h, h_{1}, h_{2} \in H, h^{\prime \prime}=h_{1}^{\prime \prime} \cap h_{2}^{\prime \prime}$ implies $h=h_{1}$ or $h=h_{2}$. Furthermore $h^{\prime \prime} \neq P$ for all $h \in H$.

$\left(\mathrm{S}_{2}^{\prime}\right)$ For all $p, p_{1}, p_{2} \in P, p^{\prime}=p_{1}^{\prime} \cap p_{2}^{\prime \prime}$ implies $p=p_{1}$ or $p=p_{2}$. Furthermore $p^{\prime} \neq H$ for all $p \in P$.

Proposition 7.1. If $M(P)$ is of finite length, then $P(H)$ is isomorphic to the partially ordered set of join- (meet-) irreducible elements of $M(P)$.

Proof. Follows immediately from the fact that $M(P)$ and $M(H)$ are dually isomorphic.

Consider now the conditions:

$\left(\mathrm{S}_{3}\right)$ For any $h_{1} \neq h_{2} \in H, q, p \in P$ such that $p \notin h_{1}^{\prime \prime}, \bar{p} \in h_{1}^{\prime \prime}$ for all $\bar{p}<p$, and $q \in h_{1}^{\prime \prime}-h_{2}^{\prime \prime}$, there exists $h \in H$ such that $q \notin h^{\prime \prime}$ and $\left(h_{1}^{\prime \prime} \cap h_{2}^{\prime \prime}\right) \cup p \subset h^{\prime \prime}$.

$\left(\mathrm{S}_{3}^{\prime}\right)$ For any $p_{1} \neq p_{2} \in P, g, h \in H$ such that $g \notin p_{1}^{\prime}, \bar{g} \in p_{1}^{\prime}$ for all $\bar{g}>g$, and $h \in p_{1}^{\prime}-p_{2}^{\prime}$, there exists $p \in P$ such that $g \notin p^{\prime}$ and $\left(p_{1}^{\prime} \cap p_{2}^{\prime}\right) \cup h \subset p^{\prime}$.

THeOREM 7.2. Let $M(P)$ be of finite length. Then $M(P)$ is modular iff $\left(\mathrm{S}_{3}\right)$ and $\left(\mathrm{S}_{3}^{\prime}\right)$ hold. 
Proof. By [8, Theorem 5], $M(P)$ is (upper) semimodular iff $\left(S_{3}\right)$ holds. By duality, $M(H)$ is (upper) semimodular iff $\left(S_{3}^{\prime}\right)$ holds. Since $M(P)$ is modular iff both $M(P)$ and its dual are (upper) semimodular, the theorem follows.

COROllary 7.3. $(P, H, I)$ is isomorphic to the incidence structure of points and hyperplanes of a finite-dimensional projective geometry $P G$ iff $(P, H, I)$ satisfies the properties $\left(\mathrm{S}_{1}\right)-\left(\mathrm{S}_{3}^{\prime}\right)$ and $M(P)(M(H))$ is of finite length.

We remark that, without any finiteness condition, in analogy with [16], the triple $(P, H, I)$ might be termed a subprojective space if $\left(\mathbf{S}_{1}\right)-\left(\mathbf{S}_{3}\right)$ and $\left(\mathbf{S}_{1}^{\prime}\right)-\left(\mathbf{S}_{3}^{\prime}\right)$ hold. However, we will not go into details.

\section{REFERENCES}

1. B. Artmann, Geometric aspects of primary lattices, Pacific J. Math. 43 (1972), 15-25.

2. R. Baer, $A$ unified theory of projective spaces and finite abelian groups, Trans. Amer. Math. Soc. 52 (1942), 283-343.

3. G. Birkhoff, Lattice theory, Amer. Math. Soc. Colloq. Publ., vol. 25, 3rd ed., Amer. Math. Soc., Providence, R. I., 1967.

4. P. Crawley and R. P. Dilworth, Algebraic theory of lattices, Prentice-Hall, Englewood Cliffs, N. J., 1973.

5. P. Dembowski and A. Wagner, Some characterizations of finite projective spaces, Arch. Math. 11 (1960), 465-469.

6. M. Dugas, Der Zusammenhang zwischen Hjelmslev-Ebenen und H-Verbänden, Universität Essen, 1977 (preprint).

7. M. Esser, Self-dual postulates for n-dimensional geometry, Duke Math. J. 18 (1951), 475-479.

8. U. Faigle, Geometries on partially ordered sets, J. Combin. Theory Ser. B 28 (1980), 26-51.

9. E. Inaba, On primary lattices, J. Fac. Sci. Hokkaido Univ. 11 (1948), 39-107.

10. B. Jónsson and G. Monk, Representation of primary arguesian lattices, Pacific J. Math. 30 (1969), 95-139.

11. W. M. Kantor, Characterizations of finite projective and affine spaces, Canad. J. Math. 21 (1969), 64-75.

12. W. Klingenberg, Desarguessche Ebenen mit Nachbarelementen, Abh. Math. Sem. Univ. Hamburg 20 (1955), 97-111.

13. __ Projektive Geometrien mit Homomorphismus, Math. Ann. 132 (1956), 180-200.

14. G. C. Kurinnoi, Condition for isomorphism of finite modular lattices, Vestnik Har'kov. Univ. 119, Mat. Meh. 40 (1975), 45-47 (Russian).

15. H.-H. Lück, Projektive Hjelmslevräume, J. Reine Angew. Math. 243 (1970), 121-158.

16. G. Markowsky and M. Petrich, Subprojective lattices and projective geometry, J. Algebra 48 (1977), 305-320.

17. J. von Neumann, Continuous geometry, Princeton Math. Surveys, no. 25, Princeton Univ. Press, Princton, N. J., 1960.

18. O. Ore, Galois connexions, Trans. Amer. Math. Soc. 55 (1944), 493-513.

19. H. Ribeiro, “Lattices" des groupes abéliens finis, Comment. Math. Helv. 23 (1949), 1-17.

20. O. Veblen and J. W. Young, Projective geometry, Vol. 1, Ginn, New York, 1916.

21. R. Wille, Primitive Länge und primitive Weite bei modularen Verbänden, Math. Z. 108 (1969), 129-136.

FB Mathematix, Technische Hochschule Darmstadt, Darmstadt, West Germany 